\title{
Qualitätssicherung in der Notfallmedizin Reanimationen im Rettungsdienstbereich Dachau
}

\author{
Ch. Günzel1 , H. Estner ${ }^{1}$, F. William¹, K. Wilhelm¹ , A. Schmuck ${ }^{2}$ \\ ${ }^{1}$ Förderverein Rettungsdienst Dachauer Land \\ 2Bayerisches Rotes Kreuz, Kreisverband Dachau
}

NOTFALLMEDIZIN 2003, 29: 159-161

Reanimationen stellen im Selbstverständnis des Rettungsdienstes einen der Schwerpunkte dar. Dabei wird die Qualität des Rettungsdienstes häufig an dieser Einsatzindikation gemessen. Viel Zeit, Geld und Personal werden investiert um Standards zu sichern und umzusetzen. Im Sinne einer nachprüfbaren Qualitätssicherung gibt es jedoch bisher kaum aussagekräftige Vergleichsdaten. Vielen regionalen Studien mangelt es an Fallzahlen oder ausgewählten Einschlusskriterien. Andererseits können große internationale Studien nicht auf örtliche kommunale Gegebenheiten übertragen werden. Durch die Erfassung eigener relevanter Daten im Rettungsdienstbereich Dachau kann ein Instrument zur Durchführung einer vergleichenden dynamischen Qualitätskontrolle vor Ort geschaffen werden.

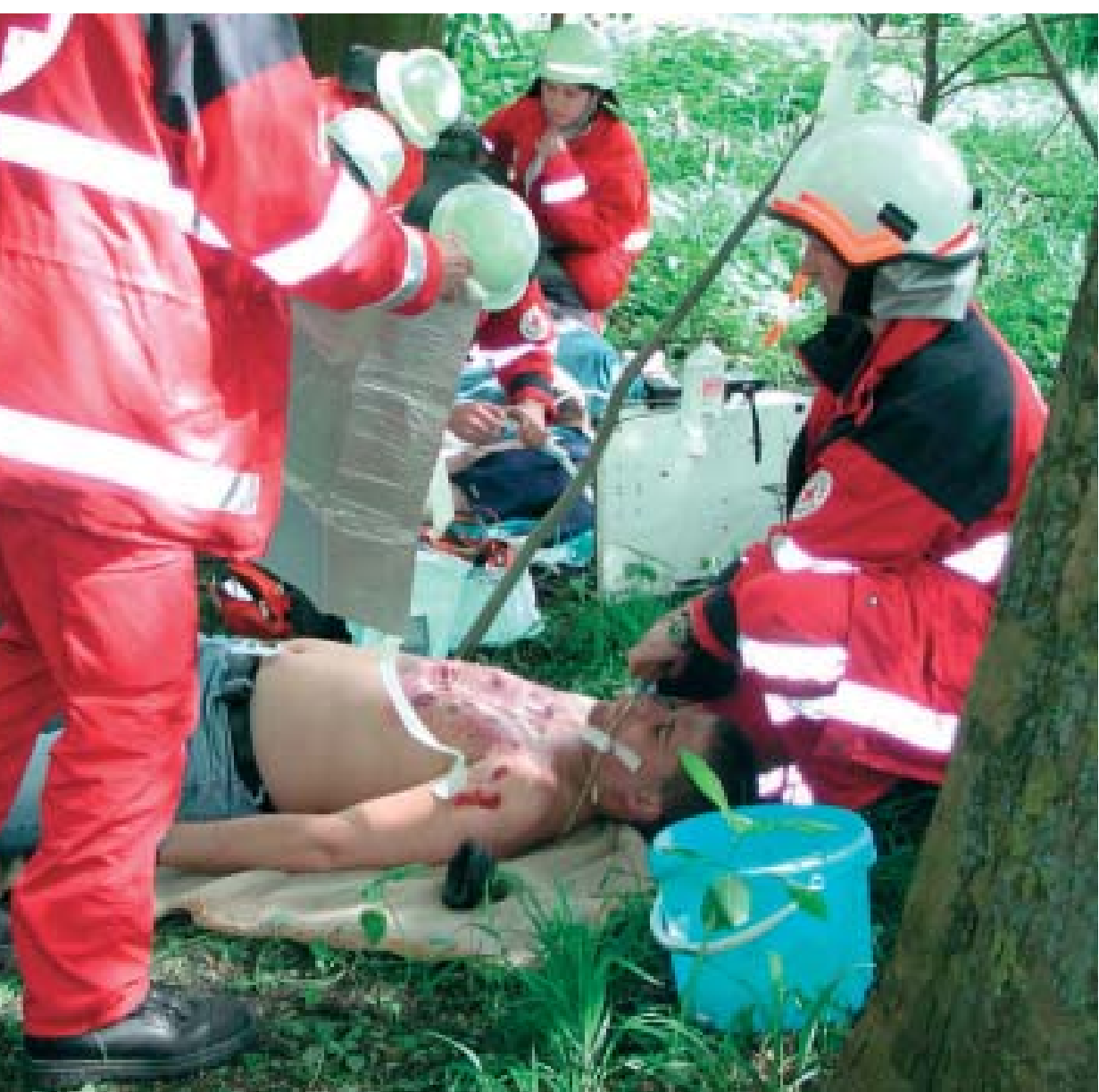

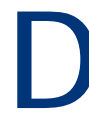
er Landkreis Dachau umfasst eine Fläche von 870 qkm mit einer Bevölkerung von insgesamt 129000 Einwohnern. Der südliche Landkreis grenzt direkt an die Landeshauptstadt München und weist eine eher großstädtische Struktur auf. Im Norden des Landkreises besteht größtenteils eine ländliche Besiedelung mit geringer Bevölkerungsdichte.

Seit dem 1. Januar 2000 werden alle präklinischen Wiederbelebungsversuche im Rettungsdienstbereich Dachau erfasst. Die folgenden Daten basieren auf der Auswertung der ersten zwei Jahre dieser Reanimationsstudie, vom 1. Januar 2000 bis 31. Dezember 2001.

\section{Rahmenbedingungen}

Der Rettungsdienst wird ausschließlich durch das Bayerische Rote Kreuz betrieben. Die mit zwei Rettungswagen (RTW) und drei Krankenwagen (KTW) besetzte Hauptrettungswache befindet sich in der Kreisstadt Dachau. Im nördlichen und westlichen Landkreisgebiet sind zwei RTW-besetzte Außenwachen eingerichtet.

Die notärztliche Versorgung wird durch zwei Notarzteinsatzfahrzeuge in Dachau und an einer Außenwache sichergestellt. Diese werden im Wechsel durch Klinikärzte und niedergelassene Ärzte besetzt. Der Landkreis liegt im Einsatzradius der Rettungshubschrauber Christoph 1 (München) sowie Christoph 32 (Ingolstadt), die bei Nichtverfügbarkeit der NEF (NotarztEinsatzfahrzeug) beziehungsweise in entlegenen Notfallorten zum Einsatz kommen. 


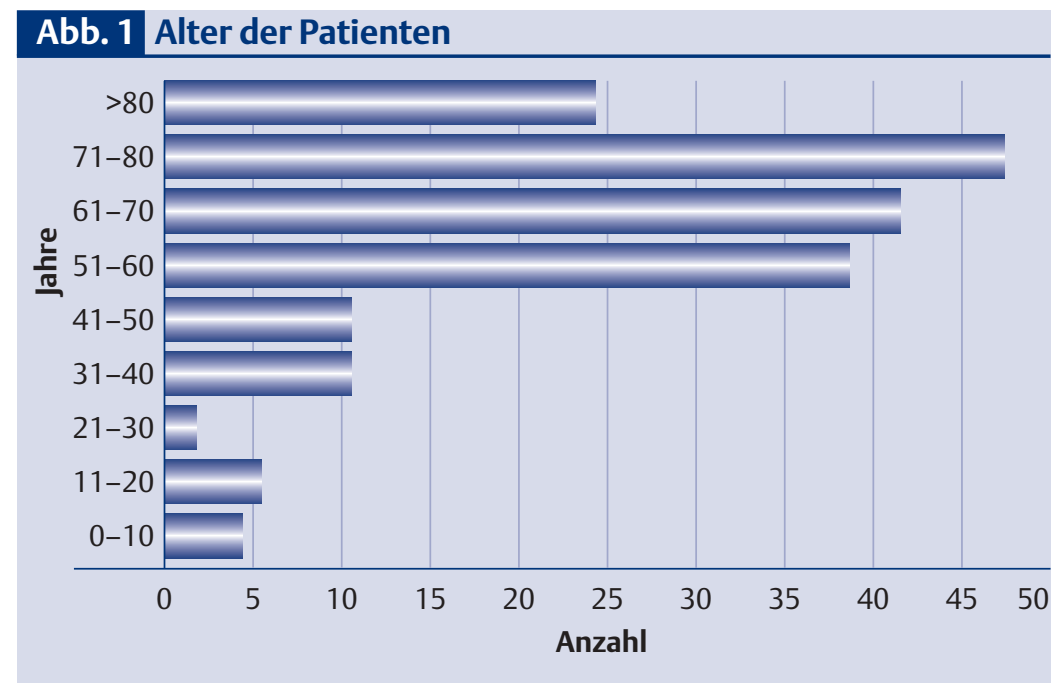

\section{Abb. 2 Einsatzzeiten}

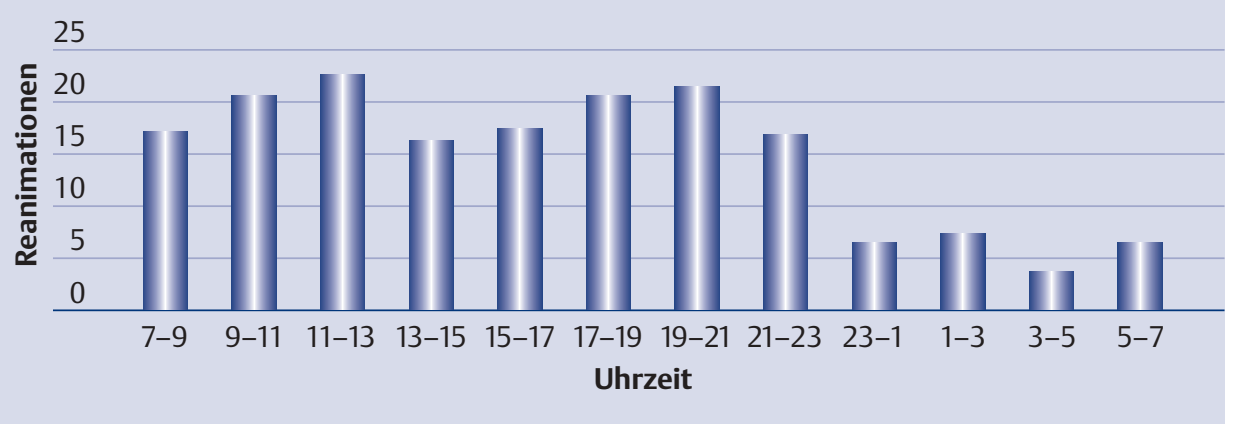

Seit mehreren Jahren wird mit großem Aufwand ein an die Einsatzfahrzeuge des BRK (Bayerisches Rotes Kreuz) gebundenes Frühdefibrillationsprojekt durchgeführt. Alle Fahrzeuge sind mit halbautomatischen externen Defibrillatoren (AED) ausgestattet; das Personal wird seit mehreren Jahren anhand von Algorhythmen in deren Anwendung geschult und in der Durch-

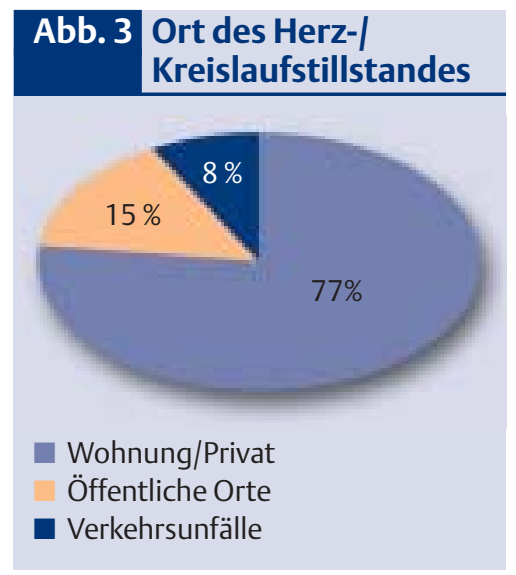

bruck, Landsberg und Starnberg. Durch diese Gegebenheiten besteht ein geschlossenes Patientengut, das ausnahmslos erfasst und ausgewertet werden kann.

\section{Datenerfassung}

In die Erfassung eingeschlossen wurden alle Fälle, in denen ein Patient einen funktionellen HerzKreislaufstillstand erlitt und mit Reanimationsmaßnahmen begonnen wurde. Es gab keinerlei sonstige Ausschlusskriterien. Diese Fälle wurden anhand eines auf den Rettungsmitteln vorgehaltenen Protokolls dokumentiert. Zusätzlich wurden alle von dem Rettungsdienstpersonal durchgeführten Maßnahmen im Rahmen der Notkompetenz in einem weiteren Protokoll festgehalten.

Anonym erfasst wurden im Protokoll patientenbezogene Daten wie Geschlecht und Alter sowie der Ort des Kreislaufstillstandes. An logistischen Daten wurden neben den eingesetzten Fahrzeugen die Alarmierungsuhrzeit, die Uhrzeit des ersteintreffenden Fahrzeugs und der Beginn der Reanimationsmaßnahmen dokumentiert. An medizinischen Daten wurde der primäre EKGRhythmus, die vermutliche Reanimationsursache und natürlich der primäre Erfolg sowie Outcome der Reanimation festgehalten. Alle primär erfolgreichen Reanimationen wurden bis zur Klinikentlassung nachverfolgt.

\section{Ergebnisse}

Im gesamten Rettungsdienstbereich Dachau gab es in den Jahren Landkreise Dachau, Fürstenfeld-
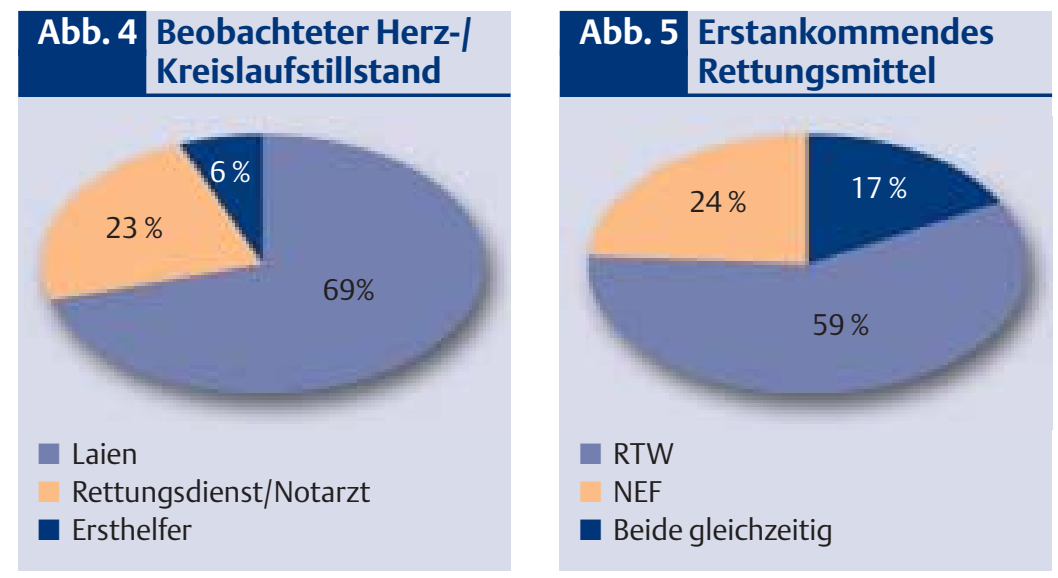
2000 und 2001 insgesamt 196 Reanimationen. Auffällig war eine signifikante Mehrzahl an männlichen Patienten (118 männlich, 78 weiblich), von $60,2 \%$. Im zweiten Jahr war eine leichte Steigerung von 90 auf 106 Reanimationen zu verzeichnen.

\section{Altersverteilung}

Der Altersgipfel lag zwischen dem 50. und 70. Lebensjahr, im Durchschnitt bei 62,3 Jahren (Abb. 1). Bei der Betrachtung nach Lebensjahrzehnten war eine deutliche Steigerung vom vierten zum fünften Lebensjahrzehnt (elf im Vergleich zu 39 Fällen) zu verzeichnen. Insgesamt wurde eine hohe Anzahl an Reanimationen im Kindes- und Jugendalter - insgesamt elf Reanimationen unter dem 20. Lebensjahr - erfasst.

\section{Einsatzzeiten}

Bezüglich der Einsatzzeiten zeigte sich ein Schwerpunkt in den Vormittags- und Nachmittagsstunden. In der Nacht zwischen 23 Uhr und 6 Uhr fanden insgesamt nur 13\% der Reanimationen statt (Abb. 2).

\section{Reanimationsmaßnahmen}

Am häufigsten fand ein Herz/Kreislaufstillstand zu Hause in der Wohnung statt (Abb. 3, Abb. 4). Es zeigte sich, dass in den meisten Fällen der RTW (59\%) vor dem NEF (24\%) eintraf. In $17 \%$ der Fälle waren beide gleichzeitig am Einsatz (Abb. 5). Aufgrund dessen kam es in 52 Fällen zur Anwendung von AEDGeräten beziehungsweise Durchführung von Notkompetenzmaßnahmen durch nichtärztliches Rettungsdienstpersonal (Abb. 6). In 27 Fällen wurde eine Frühdefibrillation bei Kammerflimmern durchgeführt, in 16 Fällen eine erfolgreiche endotracheale Intubation. In neun Fällen wurde vor Eintreffen des Notarztes Adrenalin appliziert.

Dabei begann der Rettungsdienst in über 70\% der Fälle mit den Reanimationsmaßnahmen, in nur 29,5\% begannen Laien mit der Wiederbelebung (Abb. 7).

\section{Medizinische Daten}

Als primär angenommene Ursache der Reanimation ergab sich in der Mehrzahl der Fälle eine internistische Ursache (74\%). 6\% der Fälle waren chirurgische, $18 \%$ waren unklarer oder sonstiger Genese, dabei stellten Ertrinkungsunfälle die größte Untergruppe dar (Abb. 8).

In der ersten registrierten EKGAbleitung zeigte sich bei $48,5 \%$ eine Asystolie. In 34,2\% war der primäre Rhythmus ein Kammerflimmern. In $17 \%$ lagen andere Rhythmen vor (Abb. 9).

Beim Outcome wurde unterschieden zwischen primär erfolgreichen Reanimationen mit Spontankreislauf und Transport in das Krankenhaus, sowie sekundär Überlebende von mehr als 48 Stunden. Als tertiäres Überleben galt die Entlassung aus dem Krankenhaus.

In insgesamt 80 der 196 erfolgten Reanimationen konnte eine primär erfolgreiche Reanimation verzeichnet werden, dies entspricht 40,8\% der Fälle (Abb. 10).

In den Jahren 2000/2001 konnten von 196 Reanimationen $18 \mathrm{~Pa}$ tienten die Klinik wieder verlassen (9\%). Zwölf davon waren ohne wesentliches neurologisches Defizit (Tab. 1).

\section{Zusammenfassung}

Mit der Erfassung der Reanimationsdaten wurde für den Landkreis Dachau erstmalig ein Überblick über Häufigkeit und Qualität der Reanimationsbehandlung geschaffen. Diese Basisdaten zeigen im Vergleich mit internationalen Daten ein respektables Niveau der Behandlungsqualität. Der kontinuierliche Ausbau der Ausbildung, der Aufbau eines funktionierenden Frühdefibrillationssystems und eine geregelte Freigabe der Notkompetenzmaßnahmen sind sicherlich die Grundlage der Reanimationserfolge. Jede weitere Korrektur oder Veränderung des Systems kann anhand der Veränderung der Überlebensdaten auf seine Wirksamkeit überprüft werden. Gleichzeitig kann durch die Erfassung der Daten ein sensibler Parameter geschaffen werden, um Fehler im System frühzeitig zu erfassen und Gegenmaßnahmen zu ergreifen.

Damit wurde ein wichtiger Teil der Qualitätssicherung im Rettungs-
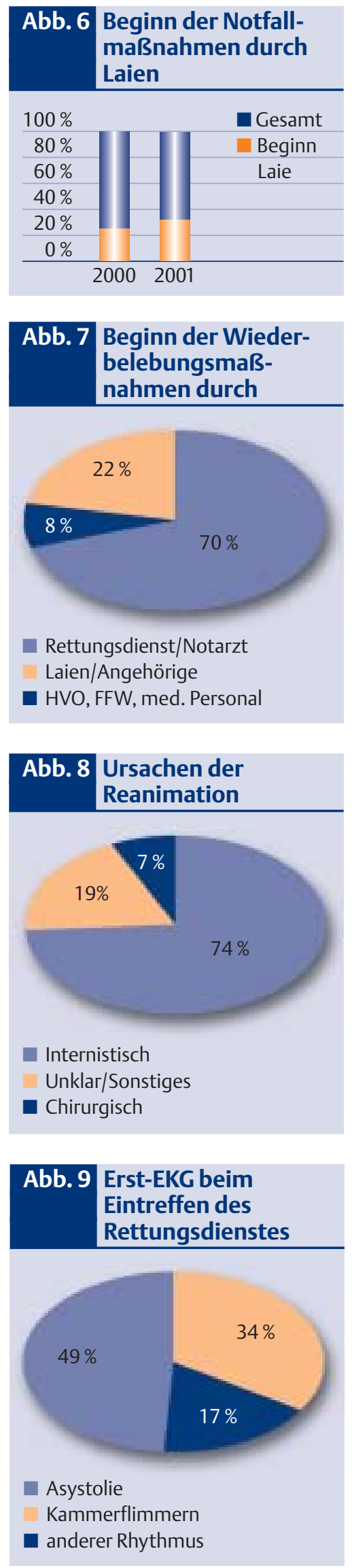
Wir wollen mehr

Informationsqualität

für Sie.

Helfen Sie mit, damit Ihnen weiterhin eine gute Fachpresse und ein optimales Informationsangebot zur Verfügung stehen.

In diesen Monaten befragt die Arbeitsgemeinschaft LA-MED Kommunikationsforschung im Gesundheitswesen e.V. Sie und Ihre Kollegen zum Leseverhalten und zu Ihren Präferenzen in der Fachpresse.

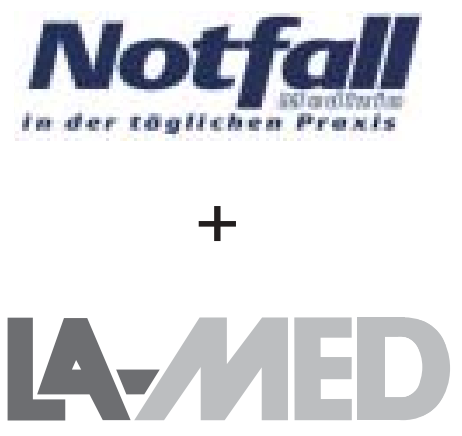

\section{Abb. 10 Primär erfolgreiche Reanimationen}

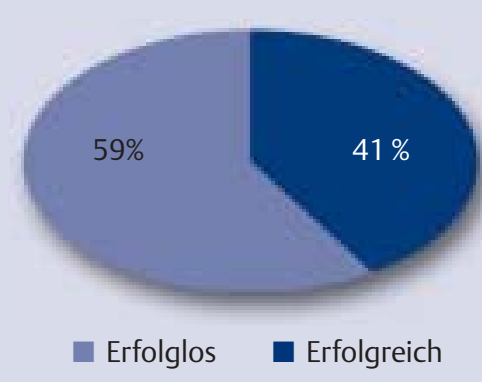

Tab. 1 Vergleich Jahr 2000 und 2001

\begin{tabular}{llll} 
& $\mathbf{2 0 0 0}$ & $\mathbf{2 0 0 1}$ & insgesamt \\
\hline Reanimationen & 90 & 106 & 196 \\
\hline $\begin{array}{l}\text { Primär erfolgreiche } \\
\text { Reanimationen }\end{array}$ & $38(=42 \%)$ & $42(=40 \%)$ & $80(=40,8 \%)$ \\
\hline Langzeitüberleben & 11 & 7 & 18 \\
\hline Frühdefi-Einsätze & 16 & 34 & 50 \\
\hline Durch HVO & 4 & 4 & 8 \\
\hline Alter (Jahre) & 61,4 & 63,0 & 62,3
\end{tabular}

dienst eingeführt. Auch hinsichtlich der in Zukunft sicher kommenden Neuerungen beziehungsweise vermeintlicher Verbesserungen im Bereich der Reanimation - wie etwa die Anwendung von AED durch Laien - wurde eine Ausgangsdatenlage geschaffen, an der sich diese orientieren und messen können.

Besonderer Dank gilt dem Rettungsdienstpersonal des BRK Dachau, ohne deren Mitarbeit die Datenerhebung nicht möglich gewesen wäre.

\section{Summary}

For the medical rescue service, reanimation is a major task, and this is often used as a yardstick to measure the quality of the service. Considerable resources in terms of time, money and personnel are invested in securing and implementing standards. With regard to quality control considerations, however, few useful comparative data have become available. Numerous regional studies lack sufficient numbers of cases or selective inclusion criteria. On the other hand, the findings of large international studies cannot be applied to the regional communal situation. By recording the medical emergency service data relevant to the Dachau area, an instrument enabling local comparative dynamic quality control can be provided.

\author{
Anschrift für die Verfasser \\ Dr. Karl Wilhelm \\ Förderverein Rettungsdienst \\ Dachauer Land e.V. \\ Münchner Straße 64 \\ 85221 Dachau \\ www.fredl.net
}

